\title{
Design of a Compact Passband Frequency Selective Surface with Stable Resonance
}

\author{
Samineni Peddakrishna, ${ }^{1}$ Taimoor Khan, ${ }^{1}$ Binod Kumar Kanaujia, ${ }^{2}$ and N. Nasimuddin ${ }^{3}$ \\ ${ }^{1}$ Department of Electronics and Communication Engineering, National Institute of Technology, Silchar, Assam, India \\ ${ }^{2}$ School of Computational and Integrative Sciences, Jawaharlal Nehru University, New Delhi, India \\ ${ }^{3}$ Institute for Infocomm Research, Singapore \\ Correspondence should be addressed to Taimoor Khan; ktaimoor@gmail.com
}

Received 2 April 2017; Revised 28 May 2017; Accepted 6 June 2017; Published 11 July 2017

Academic Editor: Xiulong Bao

Copyright (C) 2017 Samineni Peddakrishna et al. This is an open access article distributed under the Creative Commons Attribution License, which permits unrestricted use, distribution, and reproduction in any medium, provided the original work is properly cited.

\begin{abstract}
We propose a compact planar passband frequency selective surface (FSS) operating at the resonance frequency of $3.81 \mathrm{GHz}$. The proposed FSS is composed of single layer of the Archimedean spiral type with the interdigital capacitance between the corners of metallic patches printed on one side of the substrate. Compared to the operating wavelength, the size of the proposed single layer FSS is $0.066 \lambda_{0}\left(\lambda_{0}\right.$ is the free space wavelength at $\left.3.81 \mathrm{GHz}\right)$. The performance such as transmission and reflection coefficient of the proposed FSS demonstrates excellent resonance stability for different polarization and different incident angles. Finally, the optimized simulated results are validated with measured results by a fabricated prototype of $31 \times 31$ array elements on $170 \times$ $170 \mathrm{~mm}^{2}$ substrate area.
\end{abstract}

\section{Introduction}

In recent years, the wireless devices have been operating at different frequencies across the useable operating spectrum range [1]. However, in certain places, blocking the usage of some devices in favor of others may be advantageous. So, the proposal for blocking the radio waves for certain frequencies is the counterparts of the traditional filters in radio frequency (RF) circuits. In a comparison of traditional filters, the frequency selective surfaces (FSS) can perform a significant role in either stopping or passing the spectral behavior depending on the type of array element. These surfaces find a wide variety of applications such as spatial angular filters [2], frequency reuse reflector antennas [3], and radomes [4] in microwave and optics. The FSS usually consists of two-dimensional infinite extent periodic array of conducting metal patches or aperture elements on one side of the dielectric slab. The period is half of the wavelength at the resonance frequency and exhibits either transmission or reflection in the neighborhood of the element resonance [5]. However, in practical applications, FSS should be finite because it is not possible to perform infinite array characteristics with half wavelength periodic finite element FSS. To solve this difficulty, the array element should be miniaturized compared to the operating wavelength. An essential step in the design of miniaturized FSS is the proper choice of constituting array elements, substrate parameters, and the presence or absence of the FSS layers. These parameters normally control the overall frequency response of the structure, such as its resonance frequency, bandwidth, and dependence on the polarization and incidence angle. In recent years, several structures have been proposed to miniaturize the FSS [614]. Metallic patches and wire mesh separated by thin airgaps constituting an FSS [6] have been proposed at X-band. Later, in [7] the smaller element size FSS has been proposed in contrast to [6] with better resonance stability with respect to the different incident angles and polarization. A better reduction of other novel unit cells has been also proposed by adding another layer on the other side of octagonal FSS [8] and the metallic line between square patches [9]. Other novel FSS patterns have been proposed for the single band $[10,11]$ as well as dual-band [12-14], applications with stable resonance frequency at the different incident angles.

In this paper, authors have proposed a compact passband FSS with an Archimedean spiral in the center with square metal patches at the corners with the interdigital capacitance 


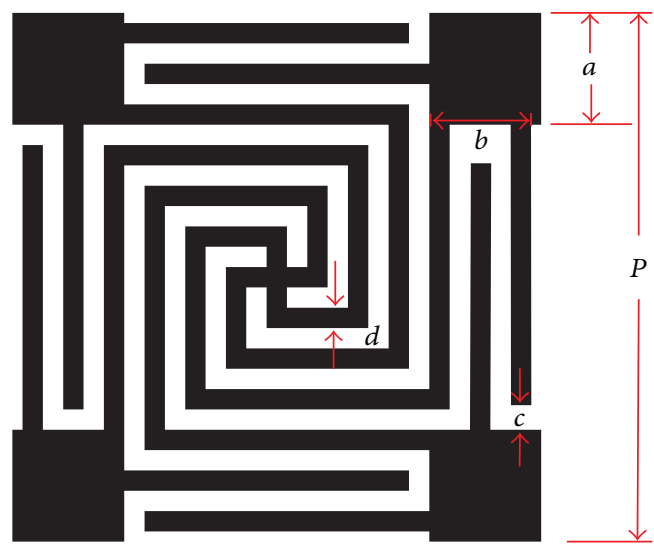

FIGURE 1: Unit cell of proposed FSS.

between them. Simulated results show that the size of FSS is $0.066 \lambda_{0}$ compared to the wavelength at the operating resonance frequency of $3.81 \mathrm{GHz}$. The proposed FSS is having excellent resonance stability for obliquely incident angles and different polarization except for a small deviation of $2 \%$ with respect to the incident angles of $60^{\circ}$ and $75^{\circ}$. Finally, the proposed FSS was fabricated with $31 \times 31$ array elements to validate the passband characteristics experimentally. Both the simulated and measured results show a good agreement. The organization of the paper is as follows: Section 2 discusses the proposed FSS unit cell and its analysis of resonance characteristics is discussed in Section 3, followed by experimental validation in Section 4. Section 5 discusses the conclusion followed by references.

\section{Proposed FSS Unit Cell}

The geometry of the proposed bandpass FSS unit cell shown in Figure 1 is designed using a low-cost FR-4 substrate $\left(\varepsilon_{r}=\right.$ $4.3, \tan \delta=0.025$, and thickness $h=1.6 \mathrm{~mm}$ ). The proposed FSS unit cell basically consists of four square metal pads at the corners along with four symmetrical spiral lines from the center and two symmetrical arrays of slots etched between the four square metal pads. The symmetrical spiral lines introduce the inductance and the gap between the square metal pads introduces the capacitance. The choice of transmission passband depends upon the size of the unit cell and the associated inductance and capacitance. Thus, a resonant frequency of the unit cell is realized from the LC circuit network. The optimized parameters of the unit cell are as follows: $P=5.2 \mathrm{~mm}, a=1.1 \mathrm{~mm}, b=1 \mathrm{~mm}$, and $c=d=$ $0.2 \mathrm{~mm}$. The black color pattern shown in Figure 1 represents the metal on the top of the substrate.

\section{Analysis of FSS Resonance Characteristics}

In order to study the characteristics of the proposed FSS, the unit cell is designed and simulated by using CST microwave studio frequency domain solver. Simulation setup is made with unit cell boundary conditions on four sides of the walls and excited with Floquet port at an arbitrary distance from top and bottom of the surface.

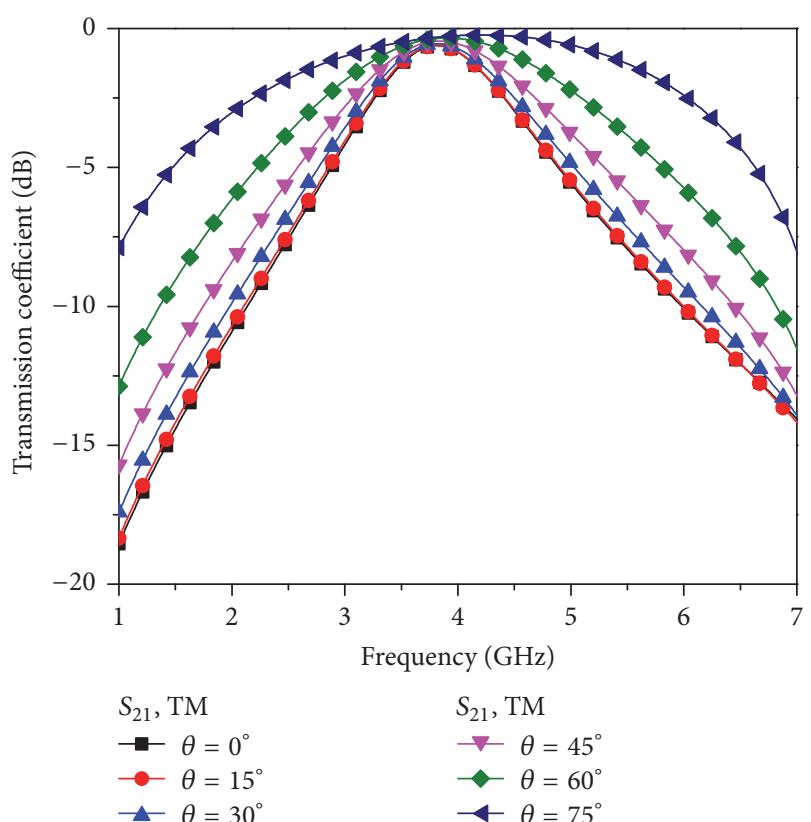

(a)

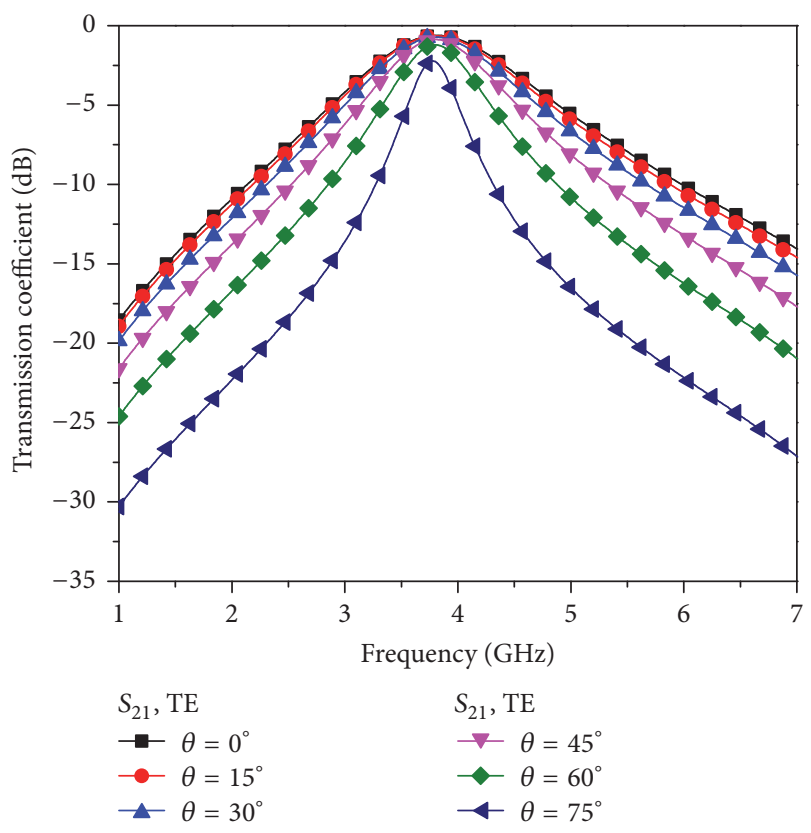

(b)

FIGURE 2: $S_{21}$ characteristics of proposed FSS under different incident angles, (a) TM mode and (b) TE mode.

The simulated transmission coefficient $\left(S_{21}\right)$ of the FSS at the different incidence angles and different polarization are shown in Figure 2. The results indicate that the structure consists of resonance stability at all incident angles and all polarization except the slight resonance frequency shift at $60^{\circ}$ and $75^{\circ}$. At these incident angles, there is a deviation of the resonance frequency of about $2 \%$. This may be due to strong surface impedance dependence at oblique incidence on the plane and polarization. However, it can also be found that the shifts of the frequency response with the change 
TABLE 1: Comparison of proposed FSS with other FSS in the literature.

\begin{tabular}{lccc}
\hline Number of metallic layers & Unit cell size & Parameters $\left(\varepsilon_{r}, h\right)$ & Operating band \\
\hline Dual [6] & $2.208 \lambda_{0} \times 2.208 \lambda_{0}$ & $3.4,0.5 \mathrm{~mm}$ & Single \\
Dual [7] & $0.104 \lambda_{0} \times 0.104 \lambda_{0}$ & $4.4,1.6 \mathrm{~mm}$ & Single \\
Dual [8] & $0.081 \lambda_{0} \times 0.081 \lambda_{0}$ & $2.65,1.5 \mathrm{~mm}$ & Single \\
Dual [9] & $0.062 \lambda_{0} \times 0.062 \lambda_{0}$ & $3.66,0.254 \mathrm{~mm}$ & Single \\
Single [10] & $0.172 \lambda_{0} \times 0.172 \lambda_{0}$ & $4.3,1.6 \mathrm{~mm}$ & Single \\
Single [11] & $0.095 \lambda_{0} \times 0.095 \lambda_{0}$ & $4.3,1.6 \mathrm{~mm}$ & Single \\
Dual [12] & $0.061 \lambda_{0} \times 0.061 \lambda_{0}$ & $2.65,1 \mathrm{~mm}$ & Dual \\
Single [13] & $0.088 \lambda_{0} \times 0.088 \lambda_{0}$ & $4.3,1.6 \mathrm{~mm}$ & Dual \\
Single [14] & $0.065 \lambda_{0} \times 0.076 \lambda_{0}$ & $2.65,1.5 \mathrm{~mm}$ & Dual \\
Structure proposed & $0.066 \lambda_{0} \times 0.066 \lambda_{0}$ & $4.3,1.6 \mathrm{~mm}$ & Single \\
\hline
\end{tabular}

$\varepsilon_{r}$ and $h$ are the permittivity and thickness of the substrate.

of the incident angle are opposite for transverse magnetic (TM) and transverse electric (TE) incidences. It is further observed from Figure 2 that the variation in the amplitude levels (i.e., the resonance bandwidth) in TM mode increases with increase in the angle of incidence, whereas in TE mode, it is falling rapidly as the angle of incidence increases. This is due to the fact that the free space TM surface impedance is proportional to the angle of incidence $\left(Z^{\mathrm{TM}}=\eta_{o} \cos \theta\right)$, whereas, in TE mode, it is opposite [15]. The resonance frequency of the passband FSS is achieved at $3.81 \mathrm{GHz}$, which is $0.066 \lambda_{0}$ at the corresponding wavelength.

Moreover, to prove the promising compactness of the proposed structure, the unit cell is simulated with available substrates with different thickness and different relative permittivity. The simulated TE-polarized transmission coefficient for normal incidence is as shown in Figure 3. It shows that the resonance frequency is shifted to $4.96 \mathrm{GHz}$ with a relative permittivity of 2.2 and thickness $h=0.762 \mathrm{~mm}$. This can also be used for WLAN applications.

\section{Experimental Validation}

To validate the characteristics of the proposed FSS experimentally, a single layer of $31 \times 31$ elements array prototype is fabricated on the FR4 substrate (size: $170 \times 170 \mathrm{~mm}^{2}$ ) and tested. The photograph of the fabricated FSS with a closed view is shown in Figure 4.

The transmission characteristics are verified under normal incidence by placing the fabricated FSS in between two horn antennas which are connected to the Anritsu N5230A vector network analyzer (VNA). The comparisons of simulated and measured results are presented in Figure 5. The simulated and measured results confirm the compactness of the proposed FSS, except a small discrepancy in the measured results. This discrepancy may be due to the fact that measurement is done on finite unit cells, whereas in simulation, the infinite array is considered by using unit cell boundary condition. Also, the fabrication tolerances due to smaller dimensions of the structure as well as environmental conditions in an anechoic chamber can also cause the deviation between the simulated and measured results.

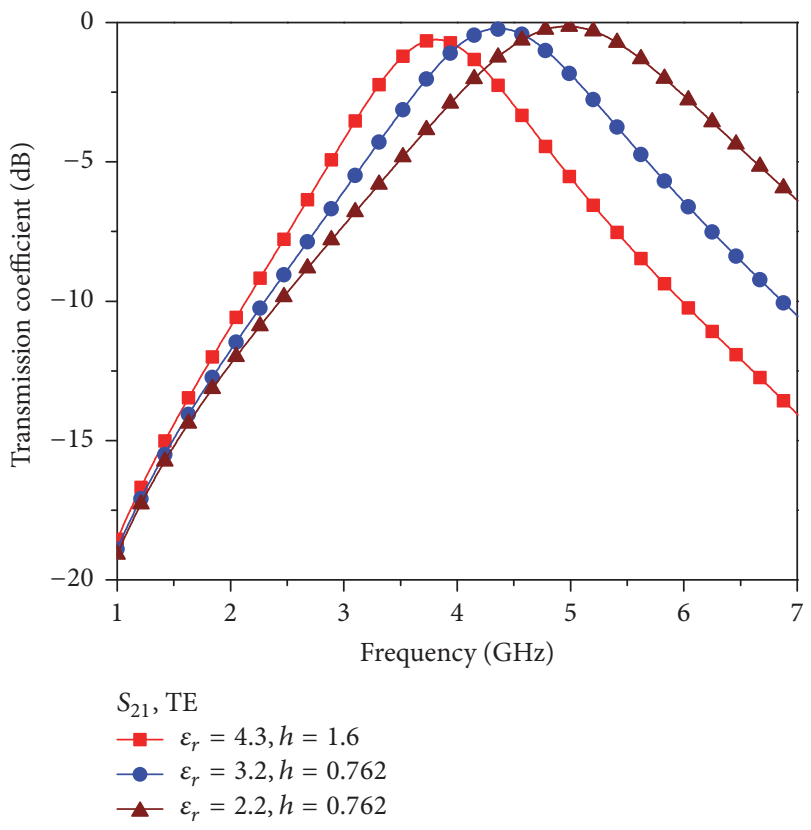

FIGURE 3: FSS characteristics of the unit cell for TE mode of polarization at different dielectric constant $\left(\varepsilon_{r}\right)$ and thickness $(h)$.

Further, the comparison of the proposed FSS with the miniaturized FSS structures available in the literature is illustrated in Table 1. Here the comparison is performed based on the metallic elements used on either single side of the substrate or dual side of the substrate with their corresponding compactness as well as number of operating bands.

\section{Conclusion}

In this paper, the authors have demonstrated a compact frequency selective surface with passband characteristics at the resonance frequency of $3.81 \mathrm{GHz}$. In addition to the compactness, the proposed FSS shows resonance stability at obliquely incidence angles with independent polarization. 


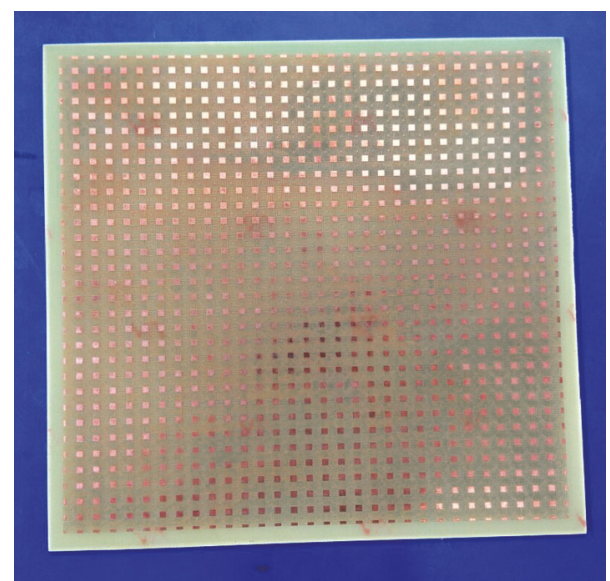

(a)

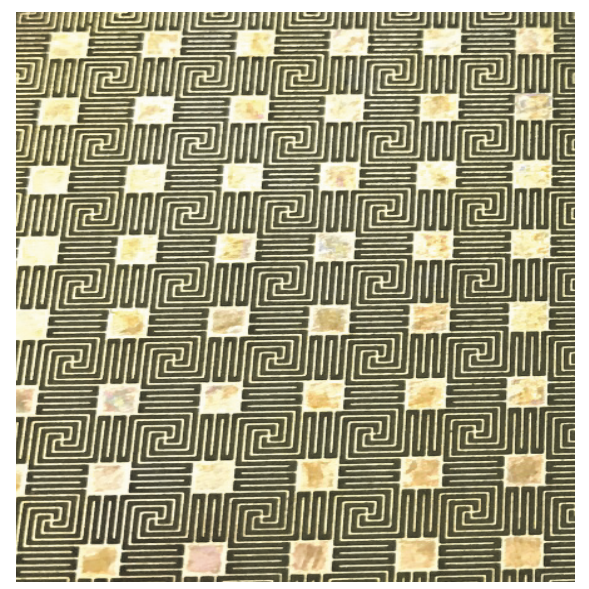

(b)

FIGURE 4: Photograph of the fabricated prototype. (a) FSS and (b) FSS zoom view.

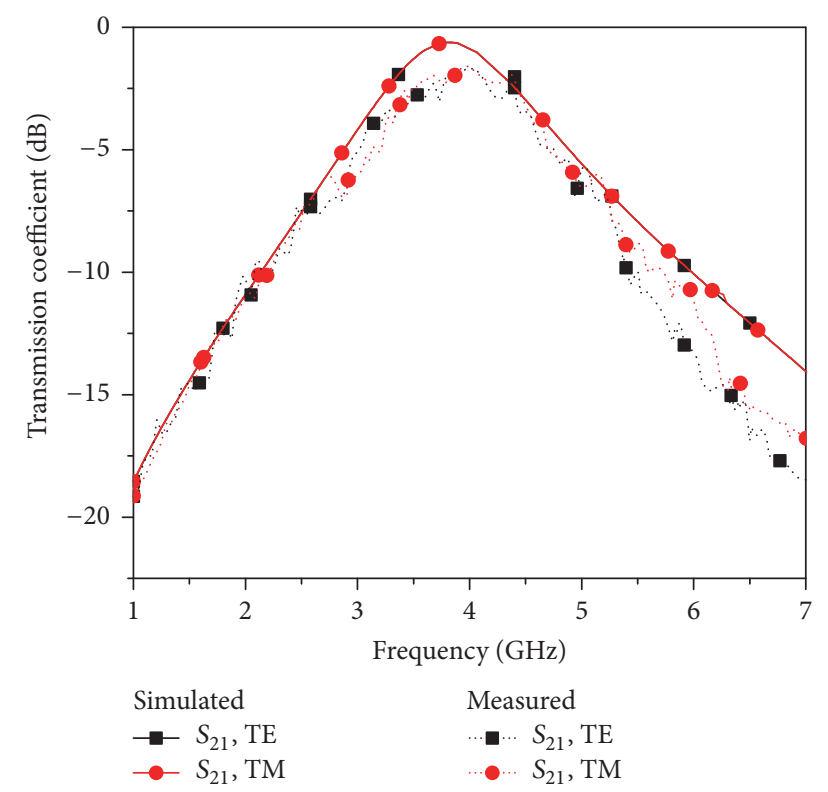

FIgURE 5: Simulated and measured transmission coefficient under normal incidence.
The characteristics were measured with a fabricated prototype of $31 \times 31$ FSS unit elements. The proposed FSS is compact and useful to reduce unwanted radiation in microwave S-/C-band fixed satellite services and WLAN.

\section{Conflicts of Interest}

The authors declare that they have no conflicts of interest.

\section{References}

[1] M. Yang and A. K. Brown, "A hybrid model for radio wave propagation through frequency selective structures (FSS)," IEEE Transactions on Antennas and Propagation, vol. 58, no. 9, pp. 2961-2968, 2010.

[2] J. D. Ortiz, J. D. Baena, V. Losada, F. Medina, and J. L. Araque, "Spatial angular filtering by FSSs made of chains of interconnected SRRs and CSRRs," IEEE Microwave and Wireless Components Letters, vol. 23, no. 9, pp. 477-479, 2013.

[3] F. O. Nians and J. Matson, "Antenna feed system utilizing polarization independent frequency selective intermediate reflector," US Patent 3-231-892, 1966.

[4] Y. J. Lee, J. Yeo, R. Mittra, and W. S. Park, "Application of electromagnetic bandgap (EBG) superstrates with controllable defects for a class of patch antennas as spatial angular filters," IEEE Transactions on Antennas and Propagation, vol. 53, no. 1 I, pp. 224-235, 2005.

[5] R. Mittra, C. H. Chan, and T. Cwik, "Techniques for Analyzing Frequency Selective Surfaces-a Review," Proceedings of the IEEE, vol. 76, no. 12, pp. 1593-1615, 1988.

[6] K. Sarabandi and N. Behdad, "A frequency selective surface with miniaturized elements," IEEE Transactions on Antennas and Propagation, vol. 55, no. 5, pp. 1239-1245, 2007.

[7] C.-N. Chiu and K.-P. Chang, "A novel miniaturized-element frequency selective surface having a stable resonance," IEEE Antennas and Wireless Propagation Letters, vol. 8, pp. 1175-1177, 2009.

[8] W. Li, T. Zhang, G. Yang, and Q. Wu, "A Novel Frequency Selective Surface with Improved Miniaturization Performance," Journal of Electromagnetic Analysis and Applications, vol. 04, no. 03, pp. 108-111, 2012.

[9] F. Deng, X. Yi, and W. Wu, "Design and performance of a double-layer miniaturized-element frequency selective surface," IEEE Antennas and Wireless Propagation Letters, vol. 12, pp. 721-724, 2013.

[10] R. Natarajan, M. Kanagasabai, S. Baisakhiya, R. Sivasamy, S. Palaniswamy, and J. K. Pakkathillam, "A compact frequency selective surface with stable response for WLAN applications," IEEE Antennas and Wireless Propagation Letters, vol. 12, pp. 718720, 2013.

[11] S. Bilvam, R. Sivasamy, M. Kanagasabai, G. N. M. Alsath, and S. Baisakhiya, "Miniaturized Band Stop FSS Using Convoluted Swastika Structure," Frequenz, vol. 71, no. 1-2, pp. 51-56, 2017.

[12] X.-D. Hu, X.-L. Zhou, L.-S. Wu, L. Zhou, and W.-Y. Yin, "A miniaturized dual-band frequency selective surface (FSS) with closed loop and its complementary pattern," IEEE Antennas and Wireless Propagation Letters, vol. 8, pp. 1374-1377, 2009.

[13] R. Sivasamy and M. Kanagasabai, "A novel dual-band angular independent FSS with closely spaced frequency response," IEEE Microwave and Wireless Components Letters, vol. 25, no. 5, pp. 298-300, 2015. 
[14] M. Yan, S. Qu, J. Wang et al., "A miniaturized dual-band FSS with stable resonance frequencies of $2.4 \mathrm{GHz} / 5 \mathrm{GHz}$ for WLAN applications," IEEE Antennas and Wireless Propagation Letters, vol. 13, pp. 895-898, 2014.

[15] O. Luukkonen, C. Simovski, G. Granet et al., "Simple and accurate analytical model of planar grids and high-impedance surfaces comprising metal strips or patches," IEEE Transactions on Antennas and Propagation, vol. 56, no. 6, pp. 1624-1632, 2008. 


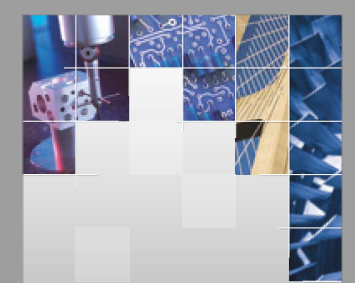

\section{Enfincering}
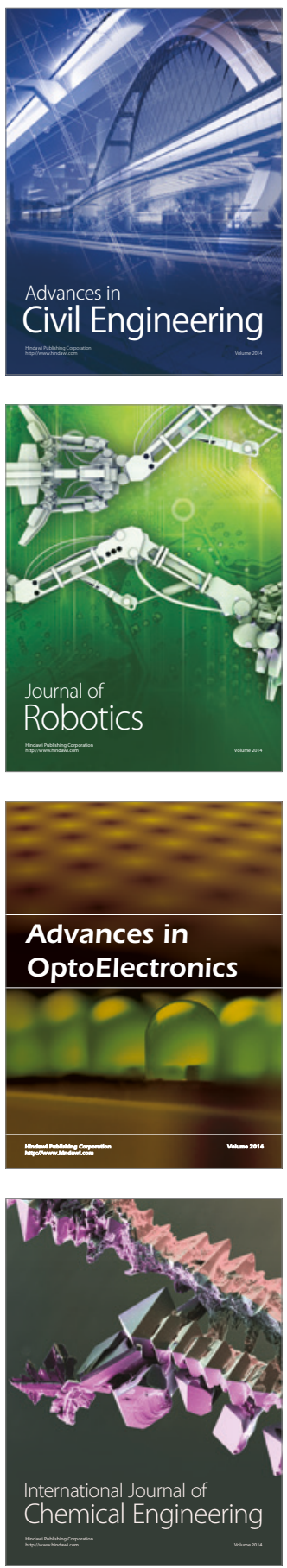

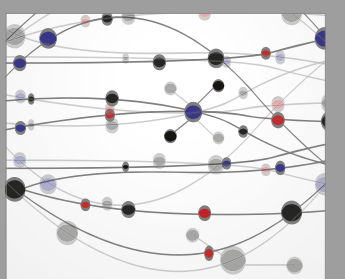

The Scientific World Journal

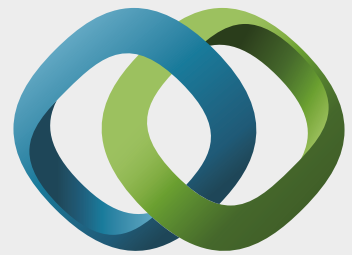

\section{Hindawi}

Submit your manuscripts at

https://www.hindawi.com
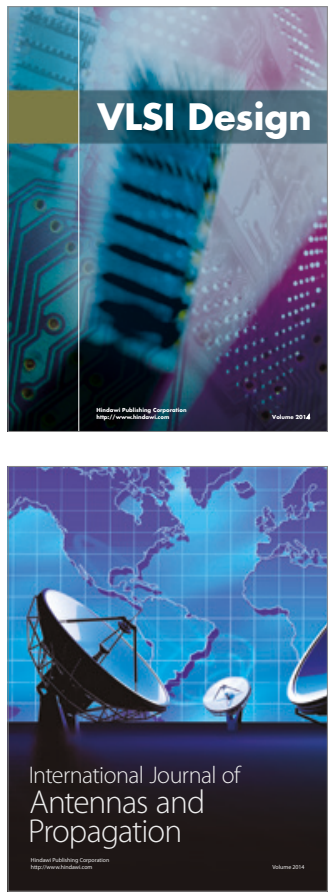

\section{Rotating}

Machinery
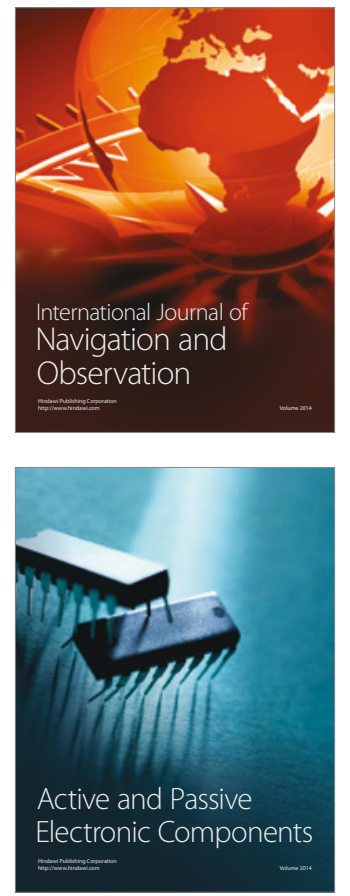
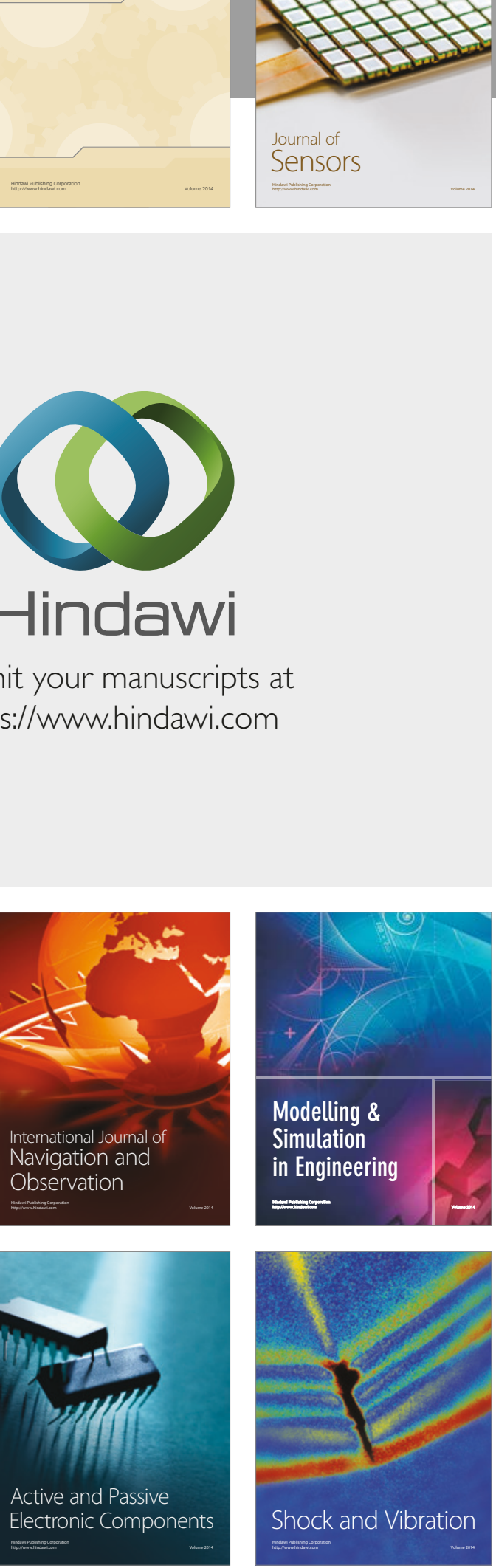
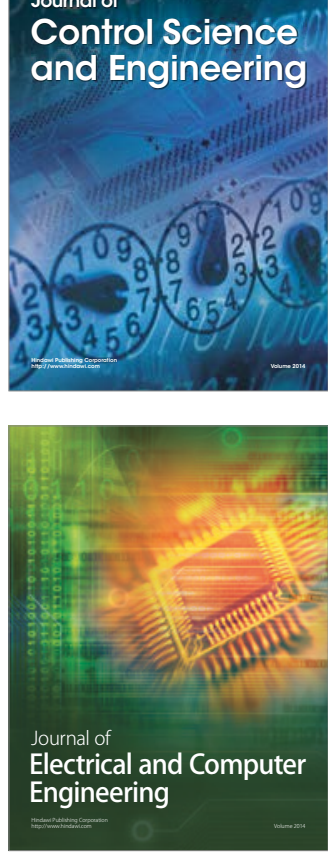

Distributed

Journal of

Control Science

and Engineering
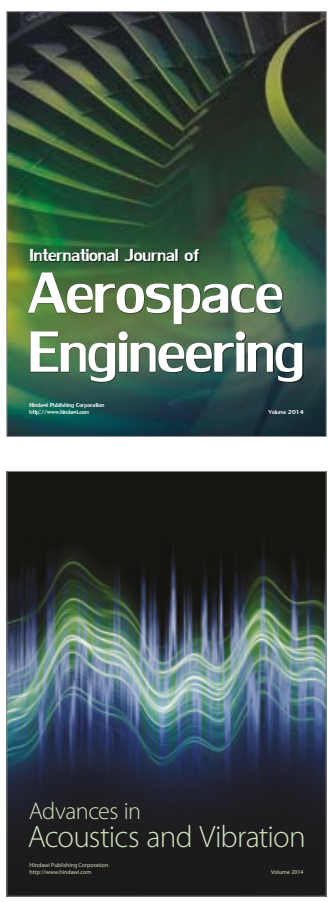

Sensor Networks 